\section{Le sol, une merveille sous nos pieds}

Feller C. (Dir.), de Marsily G., Mougin C., Pérès G., Poss R., Winiarski T., 2016, Éditions Belin, Collection Bibliothèque scientifique, 256 p. ISBN 978-2-7011-8349-7.

Cet ouvrage, rédigé à plusieurs mains par une équipe pluridisciplinaire de pédologues, agronomes, hydrogéologues, écotoxicologues, s'adresse à un large public pour lui faire prendre conscience des services que nous rendent les sols et des agressions qu'ils subissent de notre fait. Comme Eric Orsenna le souligne dans sa préface : «...triste destin que celui $\mathrm{du}$ sol, tellement inconnu alors que nous lui devons tant. ».

Effectivement, au fil de la lecture, nous découvrons que les sols sont structurés, très variables géographiquement, qu'ils hébergent de nombreuses formes de vie, qu'ils fournissent aux plantes les minéraux et l'eau indispensables à leur croissance, qu'ils alimentent les nappes phréatiques, et qu'ils subissent tant bien que mal les agressions des sociétés humaines.

Dans le premier chapitre, les auteurs aident les lecteurs à s'y retrouver dans les différentes acceptions du mot «sol» pour ses divers utilisateurs (paysans, géotechniciens) et pour les scientifiques qui s'intéressent à sa composition (minérale et organique), à sa formation à partir des roches, à sa structuration selon la profondeur (horizons organiques, d'éluviation ou d'accumulation), à sa diversité spatiale et à sa cartographie à diverses échelles.

Dans le deuxième chapitre, le lecteur découvre les rôles que joue le sol dans la nutrition des plantes et comment une théorie erronée, la théorie de l'humus d'Albrecht Thaer, a débouché sur des pratiques durables d'entretien de la matière organique du sol, alors que la théorie de la nutrition minérale de la plante, de Justus von Liebig, a promu l'usage parfois débridé des engrais chimiques dont l'efficacité est certes incontestable, mais dont les conséquences environnementales apparaissent maintenant redoutables dans les pays d'agriculture intensive. Les auteurs évoquent également la question des propriétés physiques des sols et du travail du sol. Ils dressent in fine un tableau des solutions alternatives à l'agriculture intensive conventionnelle (agriculture biologique, agroforesterie, agriculture de conservation), non sans s'interroger sur leurs domaines de validité et d'acceptabilité sociale.

Dans le troisième chapitre, le sol est présenté comme l'interface entre l'atmosphère et les nappes phréatiques. À la diversité des climats (pluviosités et températures) et des modelés géomorphologiques correspond la diversité des sols et des écosystèmes : brousse tigrée subdésertique ici, mangrove tropicale ou tourbières ailleurs. Selon les propriétés intrinsèques du sol et celles qui sont induites par les usages agricoles, les parts respectives de l'eau qui ruisselle (et érode) et de celle qui percole (et alimente les nappes phréatiques) sont très variables, et leurs conséquences environnementales sont de mieux en mieux perçues (inondations, eutrophisations des eaux continentales, prolifération des algues vertes en milieu marin, pollution par les nitrates et les pesticides des eaux domestiques). La réhabilitation du bassin d'alimentation des eaux minérales de Vittel vient heureusement donner in fine une touche optimiste à l'exposé.

Dans le quatrième chapitre, le lecteur découvre que les sols hébergent une multitude d'espèces vivantes de tailles diverses et dont la présence dans le sol peut être, soit permanente (lombrics, collemboles, nématodes, bactéries, champignons), soit temporaire (larves d'insectes, fourmis, etc.). Quantitativement, pour faire image, dans un sol tempéré, la biomasse du sol est équivalente à deux bovins ou six ovins à l'hectare. La composition de cette biomasse est très variable dans le temps au rythme des saisons (sèche ou pluvieuse, froide ou chaude) et dans l'espace en fonction de la végétation vivante et morte qui fournit les ressources trophiques (exudats, racines, litières). Dans la plupart des cas, la biodiversité de ces groupes biologiques reste à découvrir et les interactions qu'ils ont entre eux et avec les végétaux dans la rhizosphère méritent de plus amples investigations pour éviter que les systèmes naturels de biocontrôle des proliférations de pucerons, limaces, vers blancs, nématodes ne soient affectés par les interventions humaines (techniques culturales, pesticides, etc.).

Les effets positifs des amendements organiques, des prairies et des pratiques agroforestières sur les abondances bactériennes et fongiques et l'activité des lombrics dans les sols des zones tempérées permettent d'être optimiste quant à la remédiation des sols dégradés. Des méthodes nouvelles d'évaluation de la biodiversité des sols sont actuellement développées, qui permettront d'évaluer plus sûrement l'état biologique des sols et d'en faire le suivi.

Dans le cinquième chapitre sont traitées les questions de pollution des sols : pollutions métalliques liées à des activités minières ou industrielles, mais aussi agricoles pour le cuivre, le zinc, voire le cadmium; pollutions organiques par les hydrocarbures, les polychorobiphénils, les dioxines ou encore les pesticides (glyphosate, chlordecone par exemple), les antibiotiques et les hormones. Si les pollutions accidentelles ponctuelles autour des sites industriels sont faciles à identifier, les pollutions diffuses par voie atmosphérique ou par épandage de produits résiduaires (boues d'épuration, lisiers) sont plus difficiles à cerner. Soulignons que le développement mondial des grandes conurbations de plusieurs millions d'habitants ne manquera pas de poser de plus en plus de problèmes de cette nature.

Si les sols sont capables de jouer un rôle dans la gestion de ces effluents «rurbains» par adsorption sur le complexe 
argilo-humique et par transformation biologique (minéralisation, cométabolisme, stabilisation), cela dépend beaucoup de la solubilité et de la polarité des polluants et de la capacité d'échange cationique/anionique des sols (le chlordecone est adsorbé par les andosols de Guadeloupe et migre très lentement). $\mathrm{Au}$ cours de ces transformations, les produits intermédiaires ne sont pas tous moins toxiques et moins persistants et ils sont souvent plus difficiles à identifier.

Les techniques de dépollution par des plantes sont pertinentes pour les métaux lourds, mais les pollutions complexes par des produits organiques nécessitent la mise en œuvre de moyens conséquents sans doute difficiles à réunir dans des pays pauvres.

Dans le sixième chapitre sont évoqués rapidement les rapports entre les sols, la composition et la contamination des eaux et la santé humaine (tétanos, ankylostomes, ascaris, auxquels on pourrait ajouter les amibes et d'autres parasites tropicaux).

Dans le septième chapitre sont évoquées deux questions importantes: le sol comme matériau ancestral économique et écologique de construction de l'habitat et les conséquences environnementales de l'extension et de la densification des villes sur les sols et les nappes phréatiques. La surexploitation des nappes phréatiques des mégapoles littorales, combinée à une probable élévation du niveau marin, devrait à coup sûr avoir des conséquences fâcheuses comme c'est déjà le cas à Venise.

Dans le huitième chapitre, l'auteur propose au lecteur une réflexion approfondie sur l'accroissement des teneurs de l'atmosphère en gaz à effet de serre et sur le rôle que pourrait jouer le sol (au moins certains sols) comme puits de carbone. Il nous éclaire sur l'origine de l'objectif politico-médiatique d'augmenter de 4 pour mille le stock de carbone des sols de la planète.
Il souligne d'ailleurs que cet objectif n'est réaliste que si le climat permet la croissance active des végétaux et si les sols contiennent assez d'argile et de limon pour stabiliser l'humus qui en résulte (pour les sols sableux dunaires de la zone sahélienne ce sera très très dur!).

Pour éclairer le lecteur sur la complexité du processus de séquestration du carbone dans les sols, il envisage simultanément les cycles du carbone et de l'azote, car ces deux éléments sont intimement liés aussi bien dans la matière végétale vivante que dans sa transformation lors des processus d'humification. Pour évaluer les effets des pratiques culturales actuelles ou innovantes, il faut donc prendre en compte les principaux gaz qui jouent un rôle dans l'effet de serre, c'est-à-dire $\mathrm{CO}_{2}, \mathrm{CH}_{4}$, et $\mathrm{N}_{2} \mathrm{O}$. L'auteur développe deux exemples : celui de la gestion des pailles de canne à sucre lors de la récolte et celui des systèmes de culture sous couvert végétal. Il s'interroge sur la faisabilité économique et sociale de la généralisation des solutions favorisant la séquestration du carbone.

Enfin, dans le neuvième chapitre, l'auteur donne à voir aux lecteurs que les sols et leurs représentations participent au fonds culturel de nos sociétés. Cette fin, plus légère, est la bienvenue après tous ces exposés très denses et parfois inquiétants.

Après avoir lu cet ouvrage richement illustré et rédigé dans une langue facile à comprendre, vous aurez sans nul doute envie d'en savoir plus sur tel ou tel sujet: la bibliographie que les auteurs ont sélectionnée répondra sans nul doute à votre attente.

Jean-Pascal Pichot* Agronome et pédologue émérite, Les Verdiers B, 209 rue Buffon, 34070 Montpellier, France

* Auteur de correspondance : jepapichot@gmail.com 